The following resources related to this article are available online at www.sciencemag.org (this information is current as of October 14, 2009 ):

Updated information and services, including high-resolution figures, can be found in the online version of this article at:

http://www.sciencemag.org/cgi/content/full/326/5949/72

Supporting Online Material can be found at:

http://www.sciencemag.org/cgi/content/full/326/5949/72/DC1

A list of selected additional articles on the Science Web sites related to this article can be found at:

http://www.sciencemag.org/cgi/content/full/326/5949/72\#related-content

This article cites 38 articles, 6 of which can be accessed for free:

http://www.sciencemag.org/cgi/content/full/326/5949/72\#otherarticles

This article has been cited by 6 articles hosted by HighWire Press; see:

http://www.sciencemag.org/cgi/content/full/326/5949/72\#otherarticles

This article appears in the following subject collections:

Anthropology

http://www.sciencemag.org/cgi/collection/anthro

Information about obtaining reprints of this article or about obtaining permission to reproduce this article in whole or in part can be found at:

http://www.sciencemag.org/about/permissions.dtl 


\title{
Combining Prehension and Propulsion: The Foot of Ardipithecus ramidus
}

\author{
C. Owen Lovejoy, Bruce Latimer, Gen Suwa, Berhane Asfaw, Tim D. White
}

$\mathrm{T}$ he special foot adaptations that enable humans to walk upright and run are central to understanding our evolution. Until the discovery of Ardipithecus ramidus, it was generally thought that our foot evolved from one similar to that of modern African apes. Apes have feet that are modified to support their large bodies and to facilitate vertical climbing, thus allowing them to feed, nest, and seek safety in trees. Our foot differs from theirs in myriad ways, and its evolution from theirs would consequently have required an extensive series of structural changes. Some mid-20thcentury comparative anatomists were so impressed with the profound differences between human and extant ape feet that they postulated a deep, preape origin for hominids.

Ar. ramidus brings a new perspective to this old controversy. Its foot turns out to be unlike those of the African apes in many ways. The partial skeleton of Ar. ramidus preserves most of the foot and includes a special bone called the os peroneum that is critical for understanding foot evolu-
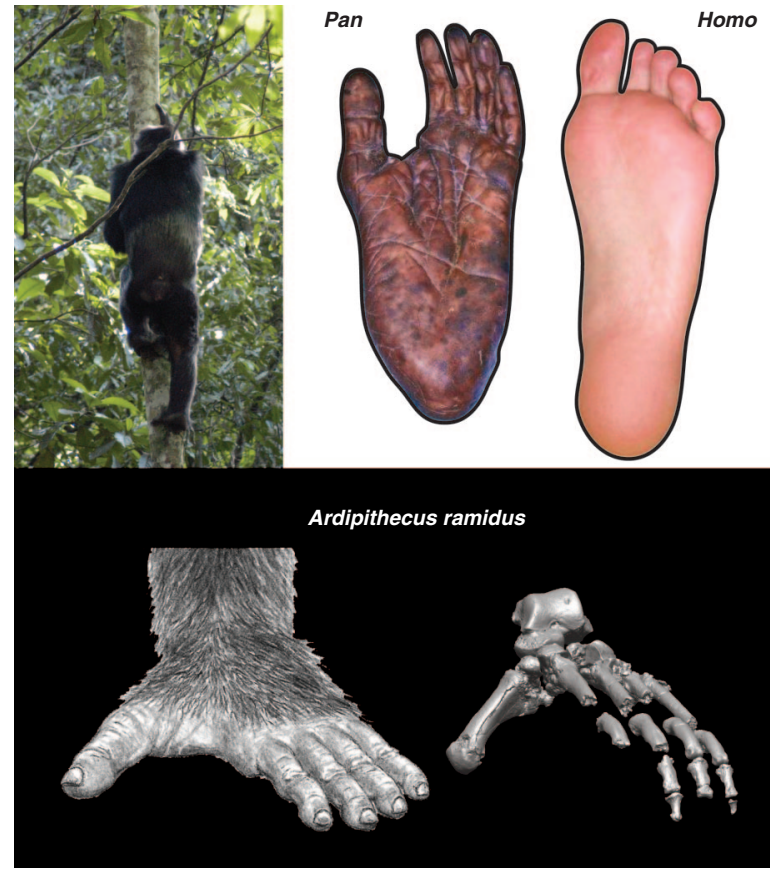

Foot skeleton of Ar. ramidus (bottom; reconstruction based on computed tomography rendering shown) lacked many features that have evolved for advanced vertical climbing and suspension in extant chimpanzees (Pan, top left). Chimpanzees have a highly flexible midfoot and other adaptations that improve their ability to grasp substrates. These are absent in Ar. ramidus. tion. This bone, which is embedded

within a tendon, facilitates the mechanical action of the fibularis longus, the primary muscle that draws in the big toe when the foot is grasping. Until now, we knew little about this bone's natural history, except that it is present in Old World monkeys and gibbons but generally not in our more recent ape relatives. Monkeys are very accomplished at leaping between trees. They must keep their feet fairly rigid during takeoff when they hurl themselves across gaps in the tree canopy; otherwise, much of the torque from their foot muscles would be dissipated within the foot rather than being transferred to the tree.

The African apes are too large to do much leaping. They have therefore given up the features that maintain a rigid foot and have instead modified theirs for more effective grasping-almost to the point of making it difficult to distinguish their feet from their hands. Indeed, very early anatomists argued that the "quadrumanus" apes were not related to humans because of their hand-like feet. Extant apes lack the os peroneum, and their fibularis tendon, which draws foot as they did: Ours turns out to have evolved in one direction, while those of African apes were evolving in quite another.

One of the great advantages of our more rigid foot is that it works much better as a lever during upright walking and running (as it also does in monkeys). However, Ar. ramidus still had an opposable big toe, unlike any later hominid. Its ability to walk upright was therefore comparatively primitive. Because it had substantially modified the other four toes for upright walking, even while retaining its grasping big toe, the Ardipithecus foot was an odd mosaic that worked for both upright walking and climbing in trees. If our last common ancestor with the chimpanzee had not retained such an unspecialized foot, perhaps upright walking might never have evolved in the first place.

When citing, please refer to the full paper, available at DOI 10.1126/science.1175832. 


\section{Combining Prehension and Propulsion: The Foot of Ardipithecus ramidus}

\author{
C. Owen Lovejoy, ${ }^{1}$ Bruce Latimer, $^{2}$ Gen Suwa, ${ }^{3}$ Berhane Asfaw, ${ }^{4}$ Tim D. White $^{5 *}$
}

Several elements of the Ardipithecus ramidus foot are preserved, primarily in the ARA-VP-6/500 partial skeleton. The foot has a widely abducent hallux, which was not propulsive during terrestrial bipedality. However, it lacks the highly derived tarsometatarsal laxity and inversion in extant African apes that provide maximum conformity to substrates during vertical climbing. Instead, it exhibits primitive characters that maintain plantar rigidity from foot-flat through toe-off, reminiscent of some Miocene apes and Old World monkeys. Moreover, the action of the fibularis longus muscle was more like its homolog in Old World monkeys than in African apes. Phalangeal lengths were most similar to those of Gorilla. The Ardipithecus gait pattern would thus have been unique among known primates. The last common ancestor of hominids and chimpanzees was therefore a careful climber that retained adaptations to above-branch plantigrady.

$\mathrm{T}$ he modern human foot is unique among mammals because it exhibits a series of adaptations that allow it to dissipate kinetic energy during foot strike in walking and running (and thus preserve its structural integrity), and to then transform into a rigid lever for propulsion during toe-off. Until now, the natural history of these adaptations has been shrouded because Australopithecus already exhibits most of them. Ardipithecus ramidus (1) now reveals much more about their evolution.

Well-preserved foot elements recovered from the Lower Aramis Member include a talus, medial and intermediate cuneiforms, cuboid, first, second, third, and fifth metatarsals, and several phalanges (2) (Fig. 1). Other Ar. ramidus foot elements are fragmentary and less informative. Here we describe these key foot elements, focusing on their implications for the locomotion of early hominids.

Talus. Hominoid tali vary extensively, limiting their value for inferring locomotor habitus (3). Even so, a deep, anteromedially projecting cotylar fossa is frequently generated by habitual tibiotalar contact during extreme ankle dorsiflexion [cartilage modeling; type 4 (4)]. Such morphology is typical of extant African apes and some Miocene hominoid taxa [e.g., $K N M-R U$ $2036 F(5)]$, but is only minimally expressed in ARA-VP-6/500-023 (Ar. ramidus) and A.L. 288las (Au. afarensis) (6).

\footnotetext{
${ }^{1}$ Department of Anthropology, School of Biomedical Sciences, Kent State University, Kent, OH 44240, USA. ${ }^{2}$ Department of Anatomy, Case Western Reserve University School of Medicine, Cleveland, $\mathrm{OH}$ 44106, USA. ${ }^{3}$ University Museum, University of Tokyo, Hongo, Bunkyo-ku, Tokyo 113-0033, Japan. ${ }^{4}$ Rift Valley Research Service, P.O. Box 5717, Addis Ababa, Ethiopia. ${ }^{5}$ Human Evolution Research Center and Department of Integrative Biology, 3101 Valley Life Sciences Building, University of California, Berkeley, CA 94720, USA

*To whom correspondence should be addressed. E-mail: timwhite@berkeley.edu
}

Trochlear geometry, absent a calcaneus or distal tibia to provide talar orientation, does not specify foot placement (7), but several characters are possible correlates of talocrural and subtalar mobility. The talar axis angle (fig. S1) (7-9) is both remarkably low and minimally variable in $A u$. afarensis and other early hominids, consistent with their stereotypically pronounced knee valgus during terrestrial bipedality (10). By contrast, this angle in $A R A-V P-6 / 500-023$ lies within the ranges of quadrupedal primates (Table 1). In addition, the flexor hallucis longus groove on the posterior aspect of the talus is both substantially more angulated and more trapezoidal in form (i.e., its superior surface is broader than its inferior), indicating a much greater range of tendon obliquity during locomotion than in A.L. 288-1, in which the groove is both more vertical and more parallel-sided (8). Together, these suggest more knee rotation during stance phase than was likely the case in Au. afarensis (10), even though the Ardipithecus pelvis implies full extension of both the knee and hip during upright gait (11). A prominent tubercle marks the presence of an anterior talofibular ligament in ARA$V P-6 / 500-023$. This landmark is absent in African apes but is usually present in Homo sapiens. However, bony evidence of local joint capsule expansion is remarkably variable (12).

Medial cuneiform and first metatarsal. $A R A$ $V P-6 / 500-088$ is a medial cuneiform (Fig. 2). Although damaged, a portion of its proximal joint surface articulates with the intact intermediate cuneiform (ARA-VP-6/500-075). The firstray metatarsal (Mt1) (ARA-VP-6/500-089) is preserved for its entire length. Its superoproximal surface is intact. This allows direct examination of first-ray abducence (Fig. 2), which was substantial and similar to that shown by extant Pan. As in African apes, the proximal Mt1 facet exhibits substantial spiral concavity for conjunct rotation on the hemicylindrical medial cuneiform facet (13). The ARA-VP-6/500 proximal
Mt1 base is therefore unlike its counterpart in Australopithecus, in which it is reniform and faces directly distally (14), indicating that it was permanently adducted $[(15,16)$; for a contrary view, see $(17,18)]$.

Cuboid. The human midtarsus is much longer than are those of extant African apes. Tarsal elongation increases lever arm length during toe-off (19-21). Elongation of the metatarsals would have also accomplished this goal, but would subject them to frequent midshaft fracture or failure of their tarsometatarsal joints [both are still common in modern humans, and their cause may be as simple as a misstep (22)]. It has been reasonably assumed that the human cuboid is highly derived from a more chimpanzee-like one for powerful plantarflexion during upright walking and running. Indeed, the eccentric placement of the modern human cuboid's calcaneal process is uniquely derived for enhanced midfoot rigidity during plantarflexion $(7,9)$.

The morphology of the African ape lateral midfoot contrasts greatly with that of humans. Their cuboids, naviculars, and lateral cuneiforms are greatly foreshortened. Associated soft tissues permit substantial laxity at their midtarsal and tarsometatarsal joints $(9,23-27)$. Such laxity facilitates plantar conformity to substrates during pedal grasping and vertical climbing $(9,28)$. However, it greatly compromises any plantarflexor torque about their metatarsal heads. The African ape cuboid's facets for the fourth and fifth metatarsals (Mt4 and Mt5) are, in addition, mildly concave, permitting such potential motion $(9,23,24)$. That such morphology is highly derived can be established by the midfoot morphology of Old World monkeys, which rely on plantarflexor torque during above-branch running and leaping - behaviors largely abandoned by great apes.

When normalized for body size, the Old World monkey cuboid is longer than are those of African apes (Table 1 and fig. S2). It is therefore notable that the $A R A-V P-6 / 500$ cuboid is equally long (Fig. 3). Moreover, its Mt4 facet is sinusoidal (suggesting immobility), and its Mt5 facet is virtually flat (also suggesting immobility). Was Ar. ramidus morphology derived for bipedality from a shortened African ape-like midfoot, or was it primitive? Resolution of this important issue is provided by another character of the Ar. ramidus midfoot that also varies strikingly among extant taxa.

The Ar ramidus cuboid exhibits an expansive facet for an os peroneum: a large sesamoid in the fibularis longus (= peroneus longus) tendon $(29,30)$. An obvious homolog is virtually constant in humans and Old World monkeys, because both taxa exhibit a constant, prominent underlying articular facet (Fig. 3). However, the os peroneum is usually cartilaginous or only partially calcified in humans, which accounts for routine reports of its absence in radiographic surveys. Both the sesamoid 
and its facet are absent in extant great apes (31).

The fibularis longus, in whose tendon this ossicle resides, performs substantially different functions in Old World monkeys, African apes, and humans. In Old World monkeys, in addition to adducting the hallux, it is also poised to prevent laxity in the cuboid, Mt4, and Mt5 joints. The mass, location, and breadth of the muscle's tendon [as judged from its contained os peroneum (Fig. 3)] suggest that it readily resists plantar cavitation of the tarsometatarsal joints, which would dissipate plantarflexor torque. In stark contrast, any supportive function in either African ape has been eliminated along with the os peroneum, and these taxa exhibit substantial midtarsal laxity even during plantigrade propulsion $(9,23-27,31)$.

In humans, the fibularis longus tendon supports the longitudinal arch and controls pedal inversion, both critical to successful bipedality [reviewed in (7-9)]. Moreover, the human fibularis longus no longer resides in the cuboid's prominent groove as it does in Old World monkeys and African apes. Instead, it (and its contained os peroneum) has become relocated more proximolaterally, outside and essentially perched above (in plantar view) the sometimes still-present cuboidal groove (32). The latter likely continues to be generated by retained

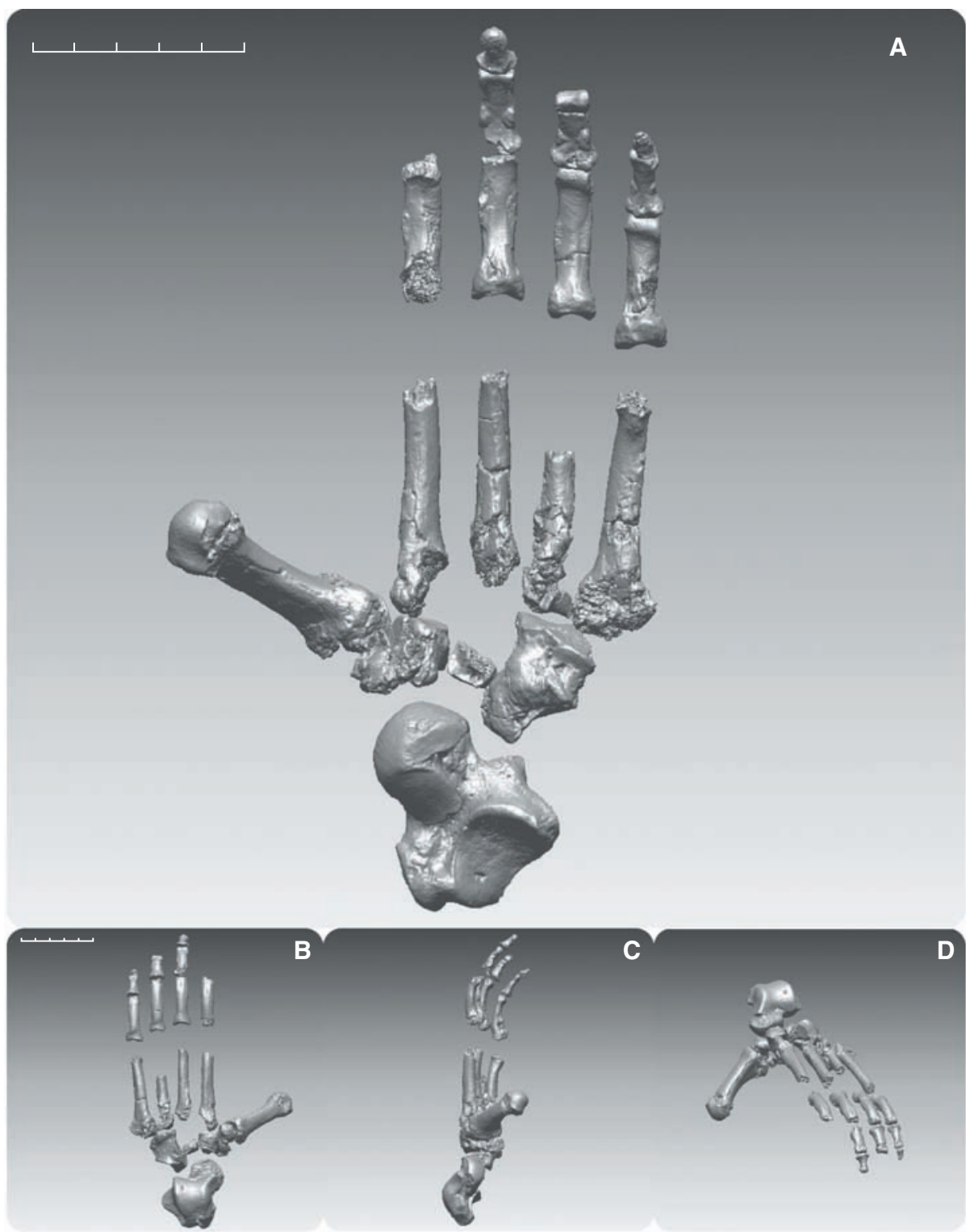

Fig. 1. Digitally rendered composite foot of $A R A-V P-6 / 500$. (A) Plantar view. (B to D) Dorsal, medial, and anteromedial oblique views, respectively. Better-preserved elements from both sides were assembled as the left foot of Ar. ramidus. Mirror-imaged elements are the talus, cuboid, Mt2 shaft, and some phalanges. The intermediate and terminal phalanges are provisionally allocated to position and side. Note the anteroposteriorly strongly abducent first ray (Fig. 2), elongate cuboid (Fig. 3), and large os peroneal facet located more distolaterally than in Homo. Cuboids of African apes generally lack an os peroneum. Scale bars, $5 \mathrm{~cm}$. Imagery is based on CT scans taken at 50 - to $150-\mu \mathrm{m}$ voxel resolution. elements of pattern formation that still underlie cuboid osteogenesis [genetically derived but selectively neutral; type 2B (4)] (33).

These are not trivial anatomical shifts in African apes (elimination of the sesamoid) or humans (relocation of the tendon's pathway). Elimination of the os peroneum in African apes, coupled with the marked anteroposterior shortening of their cuboid, causes the fibularis longus tendon to pass immediately behind and parallel to the axis of their cuboidometatarsal joints $(9,23,24)$. This allows substantial plantar conformity to the substrate even during powerful grasping of the great toe by the fibularis longus.

In contrast, translation of the tendon posteriorly in derived hominids, along with its new additional attachment to the medial cuneiform, reroutes the tendon's course so that it crosses the plantar foot more obliquely, thereby improving resistance to flexion in the cuboidometatarsal and especially the cuneiform-metatarsal joints. Both the transverse and longitudinal arches increase the tendon's moment arm to provide such resistance. Relocation of the os peroneum is thus a morphological signal of the presence of these arches. The elimination of any first-ray abduction in humans has allowed the os peroneum to vary substantially (and become merely cartilaginous), because most of the translation of the tendon has been eliminated by permanent adduction of the great toe.

Ar. ramidus morphology is clearly primitive. Its fibularis longus tendon passed over an exceptionally broad, shallow facet underlying what must have been a relatively massive os peroneum similar in size to those of most Old World monkeys (Fig. 3). Its fibularis longus could thus both adduct the great toe and plantarflex the foot, but still aid, to some extent, in support of the cuboidometatarsal joints. Not until an abducent first ray was abandoned could the os peroneum then be relocated as it is in later hominids, thereby enhancing its supportive function. It is notable, therefore, that the os peroneum facet in $\mathrm{OH}-8$ is highly derived in location and morphology (Fig. 3).

Navicular. $A R A-V P-6 / 503$ is only a small fragment of navicular, but is sufficiently preserved to further illustrate the natural history of the hominid midfoot. Despite its fragmentary condition, it suggests a primitive anteroposterior length intermediate between its homologs in extant African apes and humans. This suggests that there has been substantial midtarsal abbreviation since the common ancestor of gorillas, chimpanzees, and humans (outlined in fig. S3), and subsequent elongation of the midtarsus during hominid evolution. Indeed, a substantial portion of measurable cuboidal elongation in humans can be attributed to proximal extension of its calcaneal process, which is now located more eccentrically to further stabilize the calcaneocuboid joint during toe-off (7). Although the Ar. ramidus cuboid's calcaneal process is moderately elongate, it retained a primitive, more centroidal position. 

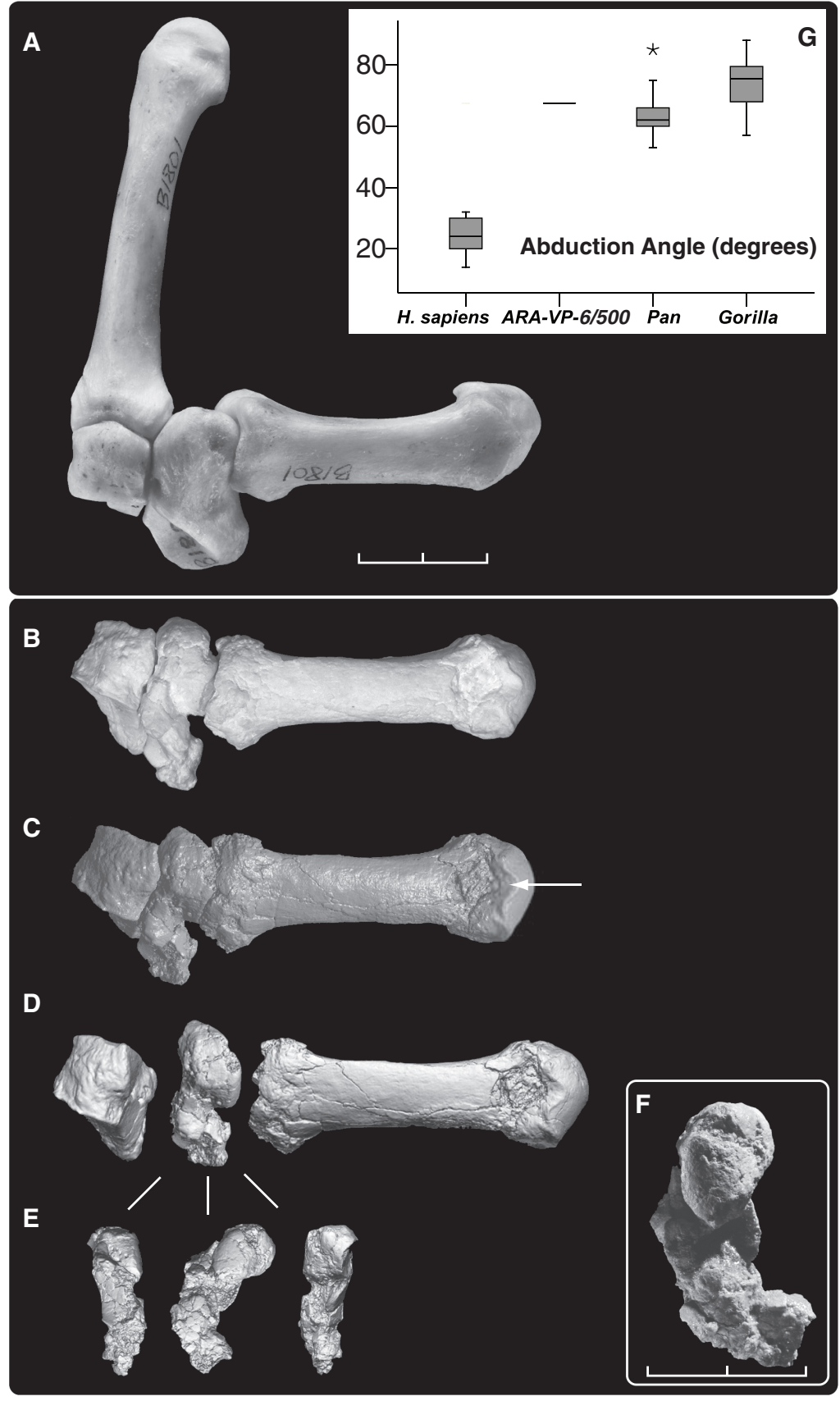

Fig. 2. First-ray abduction in Ar. ramidus. Abduction of the first ray is dependent on soft tissue structures operating about the joint, but can be readily inferred from preserved joint structure. (A) Dorsal view of female gorilla (CMNH-B1801) with Mt1 articulated with the medial and intermediate cuneiforms, showing maximum abduction without joint cavitation. (B) ARA-VP-6/500 articulated in a similar fashion (casts). Note that the two Mt1s differ in axial orientation. This difference may be a consequence of habitual bipedality in Ar. ramidus, which did not exhibit ape-like midtarsal laxity. Abduction is measured as the angle between a tangent to the distal surface of the intermediate cuneiform and the centroidal axis of the Mt1. It is $68^{\circ}$ in $A R A-V P-6 / 500$. (C and D) CT rendering of $A R A-V P-6 / 500$ in similar (C) and exploded (D) views. (E) Approximate posterior, medial, and anterior views of the medial cuneiform. (F) Medial view in dorsoplantar orientation. Although its inferior portion has suffered extensive damage, its posterosuperior portion is intact and articulates perfectly between the intermediate cuneiform and the dorsoproximal joint surface of the Mt1. Note the intact posterior portion of the plateau-like projection of the medial cuneiform's Mt1 facet distomedially. This is rare in Pan but occasional in Gorilla. Note the nonsubchondral isthmus [white arrow in (C)] separating the two articular facets on the dorsum of the Mt1. These likely record rotation of the proximal phalanx in the MP joint during grasping and terrestrial bipedality (see text). They are notably absent in $A u$. afarensis but usually present in African apes. Scale bars, $2 \mathrm{~cm}[(A)$ to $(E)], 1 \mathrm{~cm}(\mathrm{~F})$. (G) Abduction angle in $A R A-V P-6 / 500$, humans, and African apes [ $N=15$ each taxon; boxes show median, quartiles, and extreme cases in each taxon (asterisk indicates case $>1.5$ box lengths from quartile box boundary)].
Lateral metatarsals. $A R A-V P-6 / 1000$ is a right $\mathrm{Mt} 2$ lacking its head and the plantar portion of its base (Fig. 4). However, both plantar cornua are preserved, thereby permitting reasonable reconstruction of its length. The base is large. The ratio of basal height as preserved (no reconstruction or estimation) to metatarsal length lies in the upper range of Homo (fig. S4). ARA$V P-6 / 1000$ exhibits only minimal longitudinal curvature (Fig. 4) but exhibits substantial shaft torsion, which orients it for opposition with the $\mathrm{Mt1}$, as in extant African apes (fig. S5). ARA-VP$6 / 500$ lacks an intact $\mathrm{Mt} 2$, but its intermediate cuneiform also allows comparison of the joint's dorsoplantar Mt2 facet height with an estimate of body size. This ratio lies near the upper limit of the human range and outside the ranges of the African apes (fig. S6), suggesting a similarly robust base size in $A R A-V P-6 / 500$.

The Mt2's large base is readily explicable in light of its role as the foot's medial mainstay during bipedal toe-off. The dorsal edge of $A R A$ $V P-6 / 1000$ 's proximal joint surface exhibits paired chondral invaginations (Fig. 4) that are rare in the Mt2s of either gorillas or chimpanzees [one single (lateral) facet in $N=50$ ]. These cannot reflect habitual contact with the intermediate cuneiform, as this would require impossible joint cavitation. Nor does the intermediate cuneiform of $A R A-V P-6 / 500$ or any other higher primate bear matching projections; there are, instead, slight corresponding invaginations of its dorsal surface as well. Each invagination of the Mt2's dorsal surface lies just proximal to medial and lateral rugosities. In humans, these mark receipt of medial and lateral expansions of the joint's dorsal capsule [(34); this study]. Habitual, intermittent pressure against these local tarsometatarsal joint expansions almost certainly induced the paired subchondral depressions in the Aramis bone's dorsal surface. Their probable etiology [chondral modeling; type 4 (4)] is therefore informative. Substantial spiraling of the Mt2 shaft places the bone's distal end into functional opposition to the Mt1 in African apes and would have done so in $\mathrm{Ar}$. ramidus (35). Such torsion is most pronounced in the Mt 2 because it lies adjacent to the hallux, and because Mt 2 rotation is restricted by the mortising of its base between the medial cuneiform and lateral cuneiform/Mt3 laterally. The bases of the more lateral rays are less restricted and thus have (progressively) less prestructured torsion.

The developmental biology of tendon and ligament attachments is complex (36), but a markedly rugose insertion likely signals substantial Sharpey fiber investment via pattern formation (37). This is especially the case for eutherian tarsometatarsal joints, which appear to have sacrificed their proximal metapodial growth plate to encourage a more rigid syndesmosis (38). The markedly rugose tarsometatarsal joint capsule in the Ar. ramidus Mt2 suggests that it was an adaptation [direct selection acting on 
morphogenetic fields; type 1 (4)] to upright walking and running, absent any substantial loadsharing by a still abducent great toe. Moreover, the total morphological pattern of the Ar ramidus foot suggests that it exhibited a noninverted footflat during midstance [i.e., unlike that of Pan (23-27)]. The primary terrestrial role of the hallux, as in apes, would have been for balance rather than for propulsion (but see below). Powerful fulcrumation occurred only on the lateral metatarsal heads in Ar. ramidus, especially that of the Mt2, whose role in humans remains especially prominent in bipedality even after having been reinforced by the addition of a permanently adducted Mt1.

$A R A-V P-6 / 505$ is a virtually intact left Mt3 (Fig. 4). Its preserved head shows two particularly important characters. First, it exhibits dorsal doming in excess of African ape metatarsals. Second, a deep-angled gutter isolates the head from the shaft at the dorsal epiphyseal junction. Although a similar gutter is also found in ape metatarsals, it is considerably shallower, consistent with substantially less loading and excursion during metatarsophalangeal joint (MP) dorsiflexion [cartilage modeling; type 4 (4)]. Moreover, in $A R A-V P-6 / 505$, the angle between the head's dorsoplantar axis and the dorsoplantar axis of its base shows slight external torsion of the shaft, which would have optimized MP joint alignment during toe-off. This implies that growth plate loading during terrestrial bipedality predominated over that generated during grasping (i.e., it exhibits far less torsion than the Mt2, and also lacks the medial and lateral joint capsule compression facets present in ARA-VP-6/1000). The gutter also implies that loading during terrestrial bipedality was applied during substantial toe-out during and after heel-off, coupled with external rotation of the foot during late toe-off. Pro- nounced doming is entirely absent in the ARA$V P-6 / 500 \mathrm{Mt} 1$, confirming that the first ray did not participate substantially in propulsion (fig. $\mathrm{S} 12$ ). Doming is present in the Au. afarensis Mt1, again implying terrestrial bipedality with a permanently adducted great toe.

The shaft of the ARA-VP-6/505 Mt3 is only slightly curved (Fig. 4) and its base is well preserved, lacking only a minor portion of its superomedial corner. Its base morphology is remarkably similar to that of the human Mt3 in having a dorsoplantarly tall proximal articular surface (Fig. 4 and fig. S7). African ape Mt3 bases are instead regularly subdivided into distinct upper and lower portions by deep semicircular notches of their medial (for Mt2) and lateral (for Mt4) surfaces (Fig. 4 and Table 1). These serve as passageways and surfaces for tarsometatarsal and transverse intermetatarsal ligaments. The abbreviated dorsoplantar height and distinctly rhom-
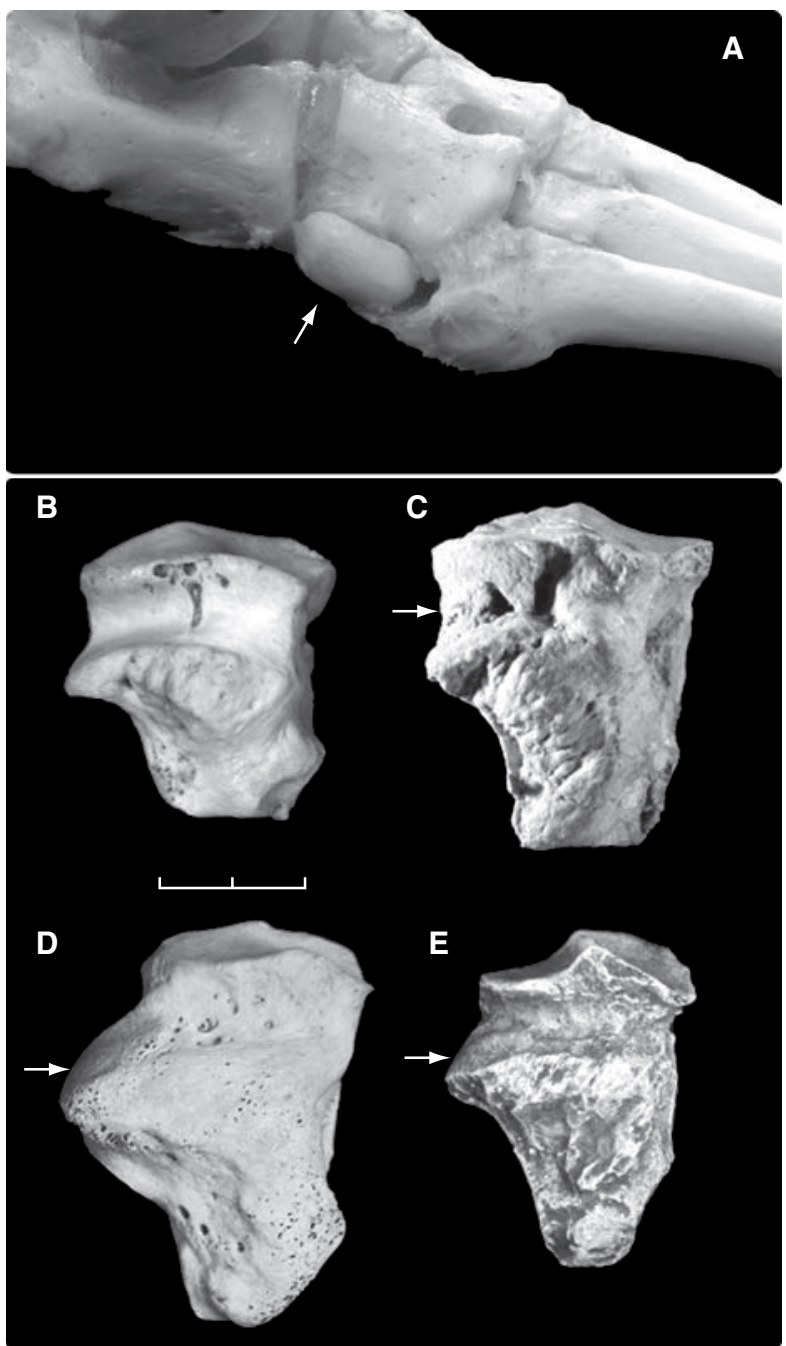

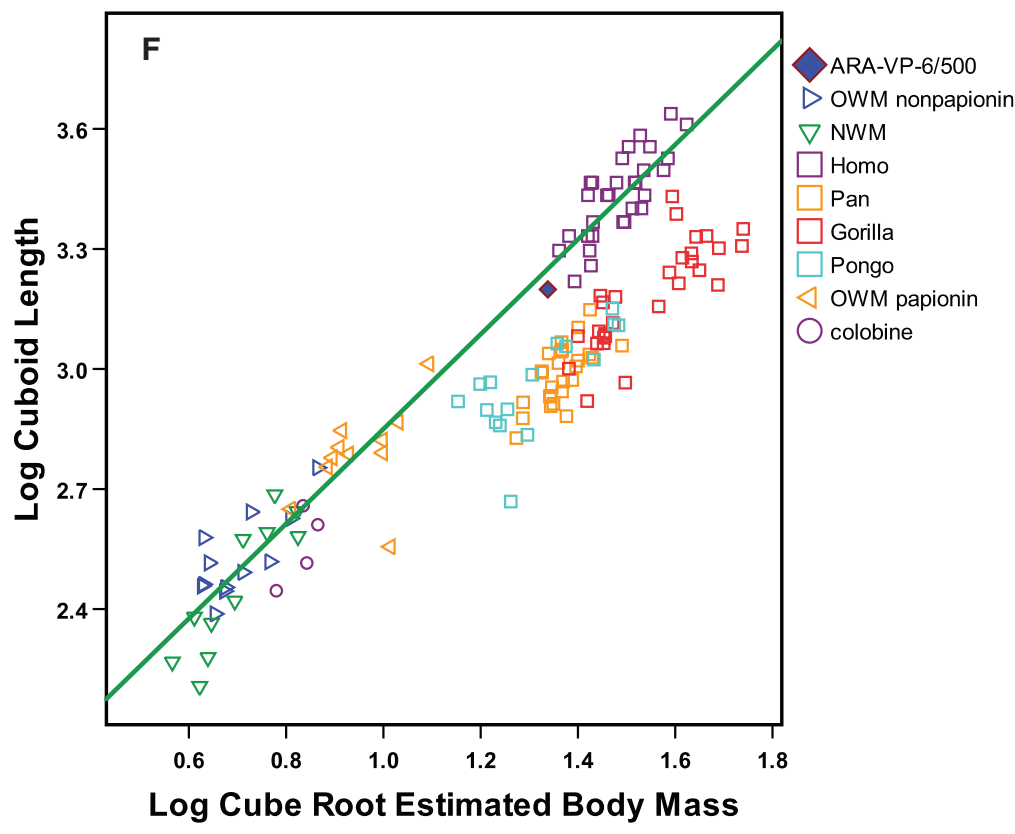

Fig. 3. Natural history of the hominoid midfoot. (A) The os peroneum. This sesamoid (white arrow in a ligamentous preparation of Papio anubis) is a large and prominent inclusion in the fibularis longus tendon of Old World monkeys, residing on an appropriately large inferolateral facet of the cuboid. In Old World monkeys, the muscle inserts at the Mt1 base, acting as both plantarflexor and hallucal adductor. Because some flexion can occur at both the calcaneocuboid and tarsometatarsal joints during climbing and terrestrial walking $(9,23,24,27)$, the fibularis longus tendon also aids plantar rigidity during plantarflexion. (B to E) Plantar surfaces of hominoid cuboids. (B) Chimpanzee (CMNH-1726). In apes, the cuboid is anteroposteriorly short and the groove in which the fibularis longus tendon lies is narrow and deep, usually with high walls. It is converted to a retaining tunnel by a homolog of the human short plantar ligament (56-58). Ape cuboids essentially lack functional os peronei [they occasionally contain small, nonfunctional, chondral bodies (31)]. (C) ARA-VP6/500-081. In Ar. ramidus the surface medial to the facet over which the tendon must pass is rugose and subperiosteal, confirming that a laterally placed os peroneum elevated its travel on the facet. (D) Human (KSU-01206). (E) OH-8 (cast; reversed). In these later hominids, the fibularis longus no longer lies in the cuboidal groove, but is instead elevated above and posterior to it by the os peroneum residing on a facet located proximolateral on the groove's proximal wall (white arrows) (32). Unlike Ar. ramidus, the fibularis longus inserts into the medial cuneiform and no longer adducts the first ray. Scale bar, $2 \mathrm{~cm}$. (F) Natural log-log scatterplot of medial cuboid length and cube root of estimated body mass in extant anthropoids (42). A regression line (reduced major axis; $y=1.184 x+1.666 ; r=0.836, N=26$ ) has been fitted to the combined cercopithecines and colobines. The most parsimonious interpretation of these data is that cuboid length in Ar. ramidus is primitive, and that the bone was elongated in later hominids (including elongation of its calcaneal process) but shortened in African apes in order to enhance hallucal grasping and plantar compliance to substrates during vertical climbing. The ranges and medians for a similar metric clarify these relationships in fig. S2. 
boidal form of African ape Mt2 and Mt3 bases [direct selection acting on morphogenetic fields; type 1 (4)] permit more plantar conformity and tarsometatarsal laxity during grasping, consistent with recent observations that the midtarsal break combines motion at both the lateral tarsometatarsal and midtarsal joints (figs. S4 and S6 to S9) $(9,23,24,27) . A R A-V P-6 / 505$ lacks this distinctive central notching morphology. This presumably reflects retention of soft tissue structures similar to those of humans. These enhance midtarsal and tarsometatarsal bending resistance from foot-flat through toe-off, ultimately culminating in the emergence of the proximal part of the long plantar ligament, which is likely derived in humans $(13,33)$. These conclusions receive strong support from geometric analysis of joint surface section moduli of the Mt3 (figs. S8 and S9).

Phalanges. Several proximal pedal phalanges from the lateral rays of Ar. ramidus preserve a base. Three are complete with proximal ends evincing clear, typically hominid dorsiflexive cants (figs. S10 and S11). Canting is more pronounced in modern humans as a consequence of the reduction of phalangeal curvature (39) (fig. S10) and abbreviation of the intermediate phalanx (figs. S12 and S13). Phalangeal shape ratios (40) are not particularly informative, but they do show that Ar ramidus phalanges are moderately robust (i.e., like those of Pan and Proconsul and unlike those of Ateles or Hylobates), with moderately deep trochleas. Midshaft robusticity is similar to that in Pan, Proconsul, and most Old World monkeys. Manual/pedal phalangeal ratios are like those in extant hominoids and unlike those in Proconsul [for discussion see $(40,41)$ ].

When complete phalanges from ray 4 of $A R A-V P-6 / 500$ are normalized by body size, their lengths fall near the Gorilla mean but below values in Pan, which may have therefore witnessed substantial phalangeal elongation since the last common ancestor of African apes and humans (fig. S13) [for further discussion, see (42)]. Pedal phalanges in Ar. ramidus are relatively shorter than those of New World monkeys (regardless of locomotor pattern), orangutans, and gibbons. Phalangeal curvature is moderate to large. The included angle of $A R A-V P-6 / 500-094$, an intact proximal phalanx of the fourth pedal ray, is $58^{\circ}$. However, its base is substantially canted, which obscures its joint angulation in lateral view. Expansion of the apical tufts of the terminal phalanges is moderate.

The first ray during terrestrial gait. The dorsal articular margin of the Mt1 head of $A R A$ $V P-6 / 500$ preserves detailed evidence of how $A r$. ramidus used its foot in some locomotor settings. Its dorsal surface bears two symmetrically placed and equal-sized V-shaped facets separated by a central nonarticular isthmus (Fig. 3). Each facet appears to have been generated by axial rotation of the ray's proximal phalanx at its MP joint [cartilage modeling; type 4 (4)].

The Mt1's dorsolateral facet was presumably generated during grasping by external rotation of both the Mt1 and its proximal phalanx, which would have brought the hallux into opposition with the lateral foot. The Mt1's dorsomedial facet would then have been generated by internal rotation that occurred when the foot was emplaced on a terrestrial substrate with the first ray in substantial abduction (because it exhibits no doming; see above). This Ar. ramidus morphology is especially notable because of its remarkable symmetry. Although similar rotation facets occur regularly on the Mtls of both Pan and Gorilla, they are most often asymmetrical and also appear to be generally deeper. In some Gorilla specimens, the medial facet is more prominent than the lateral, which suggests that during terrestrial locomotion, greater relative loads were imposed on its Mtls than in $A r$. ramidus.

This would at first seem to be a paradox, because the African apes are not habitual bipeds. However, Ar ramidus retained primitive features [a prominent os peroneum, substantial tarsometatarsal joint rigidity, a long midtarsus, and soft tissue characters that likely accompanied these (Table 2)] that allowed powerful plantarflexion about its lateral metatarsal heads, including what must have been a substantial contribution by its peroneal compartment. The African apes, by contrast, have lost such capacity in favor of substantial midtarsal laxity. This has greatly compromised the plantarflexor impulse on their lateral metatarsal heads. Partial accommodation appears to be provided by occasional or even regular impulse by their Mt1 during terrestrial gait. The Mtls of Australopithecus lack any evidence of comparable facets (15). This, and the prominent doming of their Mt1, now serve as further confirmation that the taxon lacked any first-ray abduction, and almost certainly exhibited a longitudinal arch-features that are consistent with their derived ankle morphology $(8,9,15,23,24)$ and the Laetoli footprints (43).

Interpretations and dynamics. Ar. ramidus is the only known hominid with an abducent great toe $(15,16,44)$. Its foot, along with other postcranial elements, indicates that the Late Miocene hominid precursors of $A r$ ramidus practiced mixed arboreal and terrestrial locomotion during which the lateral forefoot became extensively adapted to upright walking, even as the medial forefoot retained adaptations for arboreal exploitation.

During the gait cycle, fibularis longus contraction would also have stabilized the proximal ankle joints. The moderate to strong talar declination of the angle between the trochlea and that of the ankle's axis of rotation, in combination with clear evidence of abductor stabilization of the hip during stance phase (11), together suggest that the foot was placed near midline. The knee may have been in greater external rotation than is typical in human and Australopithecuslike (i.e., accentuated) valgus (10), with compensation by means of a more extensive range of knee rotation throughout stance phase. Ar. ramidus therefore may have lacked the consistently elevated bicondylar angle of Australopithecus.

Ar. ramidus likely relied on situationally dependent lordosis to generate functional hip abduction (minimum pelvic tilt) during stance

Table 1. Talus, cuboid, Mt1, Mt5, and Mc5 (fifth metacarpal) metrics in Ar. ramidus and other anthropoids. Values in parentheses, except for the leftmost column, denote standard deviation.

\begin{tabular}{|c|c|c|c|c|c|c|}
\hline Taxon $(N)$ & $\begin{array}{l}\text { Angle between trochlear } \\
\text { axis and talocrural } \\
\text { rotation axis }\left({ }^{\circ}\right)^{*}\end{array}$ & $\begin{array}{l}\text { Max. cuboid } \\
\text { length } \nmid / \text { body } \\
\text { size } \mp\end{array}$ & Mt1/body size $\ddagger$ & Mc5/body size $\ddagger$ & Mt5/body size $¥$ & Mc5/Mt5 \\
\hline Old World monkeys (27) & $13.2(2.2) \S$ & $1.51(0.13)$ & $4.4(0.40)$ & $4.2(0.30)$ & $6.6(0.43)$ & $0.73(0.07)$ \\
\hline New World monkeys (11) & & $1.48(0.12)$ & $4.7(0.22)$ & $4.5(0.85)$ & $6.6(0.14)$ & $0.80(0.06)$ \\
\hline Homo (30) & $10.2(2.3)$ & $1.81(0.10)$ & $3.9(0.18)$ & $2.6(0.17)$ & $4.6(0.13)$ & $0.75(0.04)$ \\
\hline $\begin{array}{l}\text { Australopithecus } \\
\text { and early Homo (12) }\end{array}$ & $7.4(1.4)$ & & & & & \\
\hline$A R A-V P-6 / 500$ & $14.5 \emptyset$ & 1.41 & 4.1 & 3.2 & 4.9 & 0.87 \\
\hline Pan (26) & $15.5(2.9)$ & $1.15(0.06)$ & $4.1(0.31)$ & $4.3(0.26)$ & $5.1(0.07)$ & $1.12(0.07)$ \\
\hline Gorilla (29) & $17.8(2.7) \|$ & $1.14(0.08)$ & $3.6(0.25)$ & $3.8(0.24)$ & $4.9(0.10)$ & $1.02(0.03)$ \\
\hline Pongo (16) & $18.4(3.5)$ & $1.18(0.16)$ & $3.6(0.22)$ & $5.3(0.39)$ & $6.7(0.11)$ & $1.03(0.05)$ \\
\hline
\end{tabular}



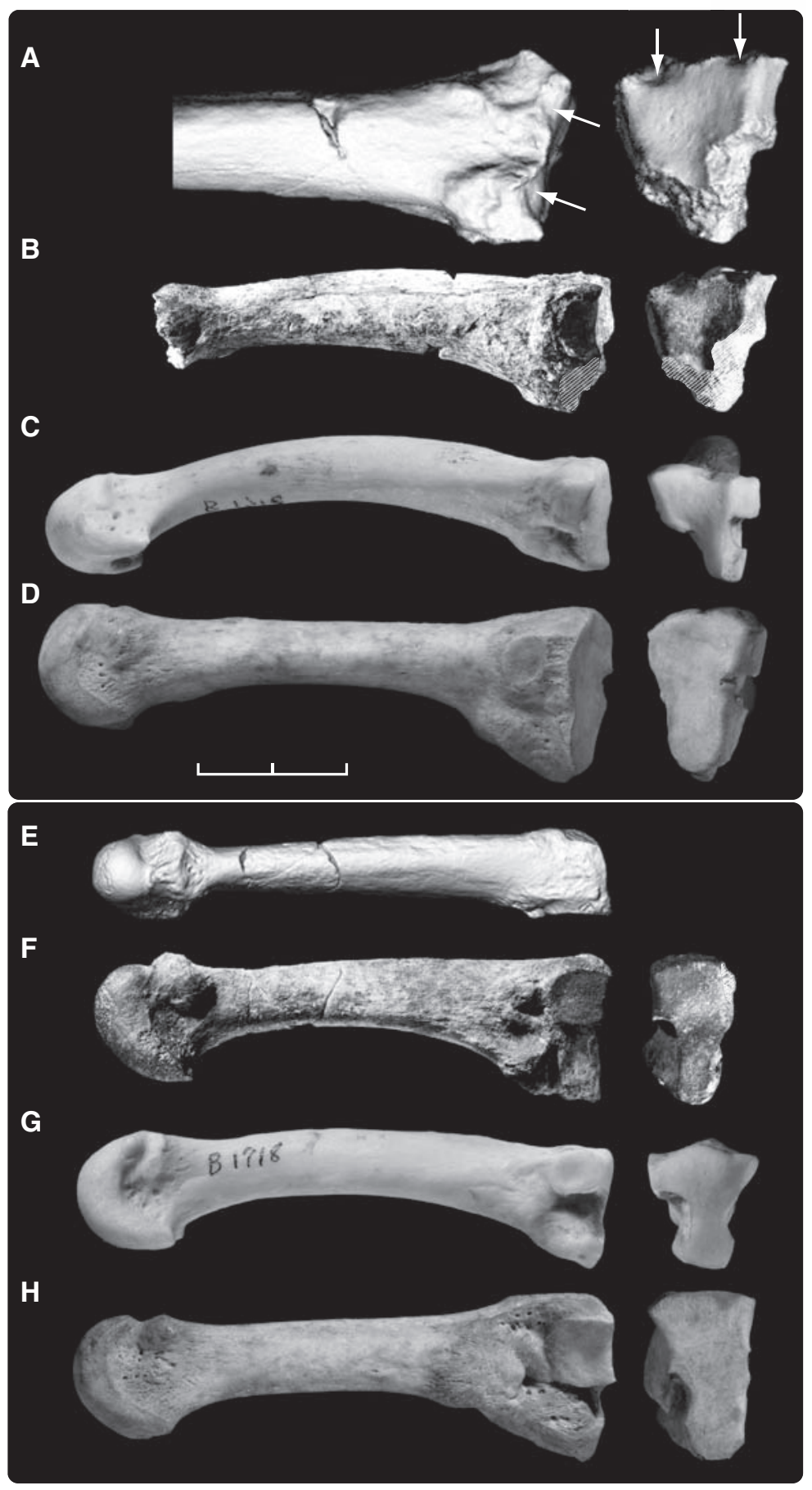

Fig. 4. Metatarsals of Ar. ramidus and extant hominoids, right Mt2 (top) and left Mt3 (bottom). (A) Enlarged $C T$ rendering of dorsal surface of $A R A-V P-6 / 1000$. Facets interpreted to be induced by rotation of its base during toe-off and grasping are indicated by arrows. These facets are shown to the right in proximal view [provided to the right in all panels except (E)]. (B) Medial view of entire original specimen (photograph). Although the head is missing, both cornua are preserved, allowing reasonable estimation of original length. Areas of postmortem damage are indicated by hatching. (C) Mt2 of Pan (CMNH-B1718). Note distinctive notching for centrally located tarsometatarsal ligaments. Damage to this area in ARA-VP-6/1000 prevents interpretation of its complete basal form. (D) Modern human Mt2. Proximal surface is superoinferiorly elongate and lacks dorsal facets, consistent with adaptation to bipedality absent an abducent great toe. (E and F) Dorsal (CT) and lateral (photograph) views of $A R A-V P-6 / 505$, an Mt3. Hatching shows minor postmortem damage. (G and $\mathbf{H}) \mathrm{Mt} 3 \mathrm{~s}$ of Pan and Homo specimens whose Mt2s are shown in (C) and (D). As is typical of the chimpanzee, the Mt3 shows bilateral notching, although it is not as pronounced in this specimen as in most. Note the striking similarity in the basal morphology of the two hominid Mt3 bases, which suggests that this morphology is likely primitive rather than derived, given the exceptionally great differences in locomotor behavior. CT methods: ARA-VP-6/ 1000, pQCT at $150 \mu \mathrm{m} ; A R A-V P-6 / 505$, microCT at $80 \mu \mathrm{m}$. Scale bar, $2 \mathrm{~cm}$. phase (11). Combined with the pedal characters described here, this suggests a form of primitive terrestrial bipedality in which the foot was emplaced at or only slightly lateral to midline, with the great toe typically in abduction (as often occurs in African apes) and the lateral forefoot in external rotation. Fulcrumation occurred along the oblique axis (fig. S12) and was obviously achieved by the triceps surae, likely substantially aided by a powerful and particularly robust fibularis longus. Balance before and during propulsion was achieved by the opposing actions of (i) a medially emplaced great toe, and (ii) plantarflexion by the fibularis longus, which would also tend to evert the foot. Thus, the lateral compartment must have been very powerful and central to its gait pattern. Indeed, the large os peroneum suggests that once the great toe was restrained by friction against the substrate, contraction of the fibularis longus could further enhance plantarflexion during heel-off through toe-off, while simultaneously maintaining rigidity in the midtarsal and tarsometatarsal joints and preventing inversion induced by substrate disconformities. At the same time, the symmetric rotary facets of the Mt1's distal joint surface, induced by MP joint motion, suggest that any eversion was prevented by a broadly abducted first ray.

Indeed, by the time of emergence of $A u$. afarensis, hominids had evolved substantially more advanced adaptations to bipedality than were present in Ardipithecus. In the former, the knee had become tibial dominant $(10)$ with accentuated valgus (exceeding even that of modern humans). Hip abduction had been established with a human-like distribution of proximal femoral cortical and trabecular bone (45-47). Moreover, in all known subsequent hominids, the more posterior location and elevation of the os peroneum facet on the cuboid [direct selection acting on morphogenetic fields; type 1 (4)] signals the presence of longitudinal and transverse arches, and thereby the addition of the transverse axis of fulcrumation (fig. S12). The facet's position in the $\mathrm{OH}-8$ cuboid is virtually human, as is the length of its calcaneal process (Fig. 2). Doming and the simpler unnotched dorsal surface of the Mt1 head characterize both $A u$. afarensis (A.L. 333-21) $(15,48)$ and Au. africanus, confirming an immobile first ray with fundamentally human-like propulsion during toe-off (43).

The feet of extant African apes are so prehensile that some early anatomists regarded them as hand homologies [reviewed and refuted in (49)]. Compared to the primitive condition of a long midtarsus as seen in taxa such as Proconsul (5), enhanced grasping required the abandonment of forceful plantarflexion on the lateral metatarsal heads in favor of increased plantar laxity at the midtarsal and tarsometatarsal joints. Primitive morphology was replaced by a shortened hindfoot and a talocrural joint modified for enhanced dorsiflexion and inversion. African apes eliminated the os peroneum, plantaris (50), and a 
Table 2. Primitive and derived states of the foot in extant taxa.

\begin{tabular}{|c|c|c|c|c|c|}
\hline \multirow{2}{*}{ Structure } & \multicolumn{5}{|c|}{ Extant state } \\
\hline & Primitive* & Old World monkey & Chimpanzee & Gorilla & Human \\
\hline Plantaris (51) & Present & Constant & $43 \%$ & Absent & $90 \%$ present \\
\hline Quadratus plantae (51) & Present & Constant & $\begin{array}{l}50 \% \text { (diminutive } \\
\text { if present) }\end{array}$ & $\begin{array}{l}29 \% \text { (diminutive } \\
\text { if present) }\end{array}$ & $\begin{array}{l}\text { Constant with novel } \\
\text { medial head }\end{array}$ \\
\hline Plantar aponeurosis & Thick and dense & Thick and dense & Minimal & Minimal & Thick and dense \\
\hline Structure and & Fused & Separate & Separate & Separate & Fused \\
\hline distribution of long & Fib. to toes $1,2,3,4,5$ & Fib. to toes $1,3,4$ & Fib. to toes $1,3,4$ & Fib. to toes $1,3,4$ & Fib. to toes $1,2,3$ \\
\hline $\begin{array}{l}\text { tibial and fibular } \\
\text { flexor tendons to } \\
\text { digits } 1 \text { to } 5(51) \dagger\end{array}$ & Tib. to toes $1,2,3,4,5$ & Tib. to toes $1,2,(4), 5$ & Tib. to toes 2 and 5 & Tib. to toes 2 and 5 & Tib. to toes $2,3,4,5$ \\
\hline $\begin{array}{l}\text { Frequency of an os } \\
\text { peroneum } \ddagger\end{array}$ & $\begin{array}{l}\text { See discussions in } \\
(29,30,55)\end{array}$ & $0.97(29)$ & $<0.04 \ddagger$ & $<0.04 \ddagger$ & $0.93+(30)$ \\
\hline Central notching & Present but with & Present but with & $86 \% \S$ & $80 \% \S$ & Absent§ \\
\hline morphology of Mt3 & inferior facets & inferior facets & No inferior facets & No inferior facets & $\begin{array}{l}\text { Usually no } \\
\text { inferior facets }\end{array}$ \\
\hline $\begin{array}{l}\text { Posterior part of long } \\
\text { plantar ligament }\end{array}$ & Probably absent & Absent & Absent & Absent & Present \\
\hline $\begin{array}{l}\text { Substantial abbreviation } \\
\text { of cuboid length }\end{array}$ & Absent & Absent & Present & Present & Absent \\
\hline
\end{tabular}

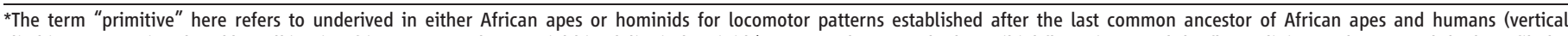

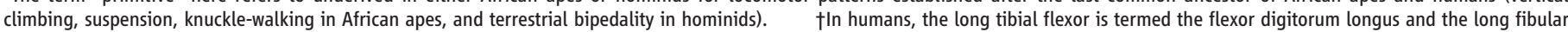

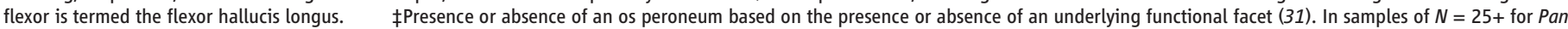
and Gorilla, one definite, human-like, facet was found in Pan [see (31)]. §Current study, $N=25$ each taxon.

functional quadratus plantae (51). This character constellation (Table 2) suggests shifts in genes encoding regulatory and signaling molecules modifying fields underlying pedal structure (52). The human plantaris is hardly functional, but its retention and association with the plantar aponeurosis as in Old World monkeys (53) signals retention of primitive features from which specialized aspects [e.g., medial head of the quadratus plantae; posterior part of the long plantar ligament (33)] could have been readily derived under selection for advanced terrestrial bipedality.

Hominid morphology has often been presumed to have evolved from ancestral morphotypes like those of extant African apes. $A r$. ramidus now establishes that this was not the case. The hominid foot was instead derived from one substantially less specialized.

\section{References and Notes}

1. T. D. White, G. Suwa, B. Asfaw, Nature 375, 88 (1995). 2. T. D. White et al., Science 326, 64 (2009).

3. C. O. Lovejoy, in Primate Morphology and Evolution, R. H. Tuttle, Ed. (Mouton, The Hague, 1975), pp. 291-326.

4. The trait nomenclature system used here is taken from $(47,54)$ and is briefly as follows [for more complete explanations, see (2)]. Type 1: traits whose morphogenesis is the direct consequence of pattern formation; usually (but not always) subject to direct selection. Type 2: traits that are genetic but are pleiotropic to, or result from hitchhiking on, type 1 traits and are not themselves subject to selection [2A: parent type 1 is inferred to be under selection but its secondary effects are not; $2 \mathrm{~B}$ : neither parent trait nor derivative is inferred to be under selection (rare)]. Type 3: resulting from a systemic growth factor. Type 4: epigenetic consequence of osteochondral remodeling and/or response to environmental stimuli, i.e., not heritable but useful in interpreting behavior. Type 5: similar to type 4, but uninformative.
5. A. C. Walker, M. Pickford, in New Interpretations of Ape and Human Ancestry, R. L. Ciochon, R. S. Corruccini, Eds. (Plenum, New York, 1983), pp. 325-413.

6. D. C. Johanson et al., Am. J. Phys. Anthropol. 57, 403 (1982).

7. V. T. Inman, The Joints of the Ankle (Williams and Wilkins, Baltimore, 1976).

8. B. Latimer, J. C. Ohman, C. O. Lovejoy, Am. J. Phys. Anthropol. 74, 155 (1987).

9. ]. M. DeSilva, Proc. Natl. Acad. Sci. U.S.A. 106, 6567 (2009).

10. C. O. Lovejoy, Gait Posture 25, 325 (2007).

11. C. O. Lovejoy et al., Science 326, 71 (2009).

12. C. O. Lovejoy, K. G. Heiple, Nature 235, 175 (1972).

13. 0. ]. Lewis, Functional Morphology of the Evolving Hand and Foot (Clarendon, Oxford, 1989).

14. Schultz noted that the medial cuneiform-Mt1 joint is "directed more forward and less sidewise in the mountain gorilla than in the other apes" [(20), p. 395]. Our sample contained only "western" specimens (G. g. gorilla).

15. B. Latimer, C. O. Lovejoy, Am. J. Phys. Anthropol. 82, 125 (1990).

16. H. M. McHenry, A. L. Jones, J. Hum. Evol. 50, 534 (2006).

17. R. J. Clarke, P. V. Tobias, Science 269, 521 (1995).

18. K. D. Hunt, J. Hum. Evol. 26, 183 (1994).

19. A. H. Schultz, Symp. Zool. Soc. London 10, 199 (1963).

20. A. H. Schultz, Hum. Biol. 2, 303 (1930).

21. A. H. Schultz, Folia Primatol. 1, 150 (1963).

22. J. T. Campbell, L. C. Schon, in Orthopaedic Surgery: The Essentials, M. E. Baratz, A. D. Watson, ]. E. Imbriglia, Eds. (Thieme, New York, 1999), pp. 591-614.

23. J. M. DeSilva, thesis, University of Michigan (2008).

24. J. M. DeSilva, Am. J. Phys. Anthropol. 10.1002/ ajpa.21140 (2009)

25. H. Elftman, J. Manter, Am. J. Phys. Anthropol. 20, 69 (1935).

26. H. Elftman, J. Manter, J. Anat. 70, 56 (1935).

27. E. Vereecke, K. D'Aout, D. De Clercq, L. Van Elsacker, P. Aerts, Am. J. Phys. Anthropol. 120, 373 (2003).

28. J. G. Fleagle et al., Symp. Zool. Soc. London 48, 359 (1981).

29. T. Manners-Smith, J. Anat. 42, 397 (1908).

30. J. M. Le Minor, J. Anat. 151, 85 (1987).

31. In two Pan specimens dissected for this paper, a small cartilage nodule (invisible upon $x$-ray) could be palpated within the tendon but had no effect on its caliber, nor was either accompanied by a facet. This accounts for reports by some earlier anatomists that the sesamoid is present in African apes (although it is clearly present in gibbons, which exhibit regular facets). A recent review (55) concluded an incidence of $2 / 11$ for the os peroneum in apes from classical literature. However, this datum is likely unreliable, because many early authors did not inspect the tendon closely and typically reported its structure "as in humans," whereas "the few anatomists who have explicitly looked for the [os peroneum] have noted its absence in Gorilla..., Pan troglodytes..., and...Pongo pygmaeus" [(30), p. 93]. Examination of the cuboid is the most reliable standard because a functional os peroneum cannot obtain without an underlying facet, just as the presence of a clearly functional facet assures the ossicle's presence, whether or not it was calcified. In rare cases, a facet-like discoloration of the bottom of the fibularis longus groove in Pan can be seen. However, the depth and cylindrical nature of the groove make such facets (if in fact they supported an os peroneum during life) largely nonfunctional, and we here report only clearly functional facets. One such human-like facet was found in a Pan specimen in our survey. Interestingly, its associated Mt4 and Mt5 facets suggested hypermobility at these joints far in excess of other Pan specimens. This supports the argument that relocation of the tendon, as in humans, does in fact reduce general midtarsal mobility.

32. M. A. Edwards, Am. J. Anat. 42, 213 (1928).

33. In primates, a fascial sheet spans the plantar aspect of the foot from calcaneus to cuboid, lateral cuneiform, and lateral metatarsal bases, and underlies the fibularis longus tendon in its course to the first ray. In humans this sheet is described as two separate elements, the short and long plantar ligaments. However, in apes the fascial sheet bridges the cuboidal groove, transforming it into a tunnel confining the fibularis longus tendon $(56,57)$. Old World monkeys have a rough equivalent but retain an os peroneum lateral to the tunnel. In humans, the fibularis longus tendon lies outside (plantar to) the cuboidal groove, and the short plantar ligament terminates proximal to it. A second portion of the sheet, which lies plantar to the tendon, spans it and inserts distally on the lateral metatarsal bases. In humans this is distinguished as the long plantar ligament $(57,58)$. These human divisions of the plantar sheet are therefore likely derived (13). 
34. R. J. Terry, in Morris' Human Anatomy, ]. P. Schaeffer, Ed. (Blakiston, Philadelphia, 1942), pp. 266-376.

35. We base this simply on torsion of the bone's diaphysis because its head was not preserved. We have not provided a numerical value because estimating the articular head's dorsoplantar axis in near-adult (but still unfused) African ape Mt2 growth plates provides only a broadly reliable estimate of the exact angle between the proximal and distal joint axes when the (unfused) head is articulated on its growth plate.

36. X. Chen, C. Macica, A. Nasiri, S. Judex, A. E. Broadus, Bone 41, 752 (2007)

37. A. Zumwalt, J. Exp. Biol. 209, 444 (2006)

38. P. L. Reno, W. E. Horton Jr., R. M. Elsey, C. O. Lovejoy, J. Exp. Zool. B 308, 283 (2007).

39. Considerable debate has centered around the importance of phalangeal shaft curvature in Au. afarensis, largely with respect to its etiology. If developmentally plastic [cartilage modeling; type 4 (4)], then curvature may imply active grasping history. However, if a direct product (or pleiotropic effect) of positional information [types 1 or 2 (4)], then it can be moot with respect to phalangeal function in rapidly evolving species. The fact that curvature increases during maturation does not resolve this issue because increasing curvature during growth is as explicable by positional information as it is by hypothetical strain regimen(s). Current evidence, such as the phalangeal curvature in great ape fetuses, supports a type 1 status. An important element that has long been ignored is phalangeal morphology in $A u$. afarensis itself (A.L. 333-115); see fig. S11.

40. D. R. Begun, M. F. Teaford, A. Walker, J. Hum. Evol. 26 89 (1994).

41. M. Nakatsukasa, Y. Kunimatsu, Y. Nakano, T. Takano, H. Ishida, Primates 44, 371 (2003)

42. C. O. Lovejoy et al., Science 326, 70 (2009).

43. T. D. White, G. Suwa, Am. J. Phys. Anthropol. 72, 485 (1987).

44. STW-573 ("Little Foot") preserves a talus, navicular, medial cuneiform, and Mt1. Its first ray has been described as partially abducent $(17,18)$. Replacement of its talus and/or navicular with those of either a chimpanzee or human would have had no substantial effect on first-ray abducence; they are not informative. The higher primate medial cuneiform itself is not mobile and is therefore equally uninformative, save for its Mt1 joint surface. This surface faces distally, is virtually flat, and is therefore immobile in STW-573. The claim that "the first metatarsal facet overflows from distal to proximal, as in apes" [see footnote 18 of (17)] is incorrect, and the dotted line drawn to indicate the proximal extent of the facet in their figure $3 \mathrm{~B}$ is exaggerated. The joint surface of STW-573 is virtually identical to that of $\mathrm{OH}-8$ and unlike that of any ape. This alone falsifies the contention that the specimen's hallux was abducent. Indeed, the fibularis longus insertion of STW-573 is described as spanning both the medial cuneiform and Mt1 and therefore cannot have adducted the Mt1 even if it retained any mobility in the first tarsometatarsal joint.

45. C. O. Lovejoy, Gait Posture 21, 95 (2005).

46. C. O. Lovejoy, Gait Posture 21, 113 (2005).

47. C. O. Lovejoy, R. S. Meindl, ]. C. Ohman, K. G. Heiple, T. D. White, Am. J. Phys. Anthropol. 119, 97 (2002).

48. C. V. Ward, Yearb. Phys. Anthropol. 119S-35, 185 (2002).

49. T. H. Huxley, Evidence as to Man's Place in Nature (Williams and Norgate, London, 1863).

50. The plantaris is "normally present in prosimians, monkeys, and man, but is lacking in gibbons and gorillas, nearly all orang-utans, and a considerable percentage of chimpanzees" (53).

51. W. L. Straus Jr., Q. Rev. Biol. 24, 200 (1949)

52. Interpretation of structure frequency requires constant attention to the selective mechanism in play. Narrowly defined (i.e., named) anatomical structures (e.g., long and short plantar ligaments) emerge from mesenchymal fields, which are manifestations of their parent positional information. It is therefore field configuration that is the target of selection. Constant structures within a species (e.g., the Achilles' tendon) suggest low field variance and intense stabilizing selection. Conversely, substantially reduced frequencies of a "named" tissue mass within a species (e.g., the quadratus plantae in Pan) signal the occurrence of underlying field shifts. These may emanate from selective encouragement of changes in target structures that share field commonality with the reduced structure, simple relaxation of selection, selection against the structure, or some combination of these.
53. C. G. Hartman, W. L. Straus, The Anatomy of the Rhesus Monkey (Williams and Wilkins, Baltimore, ed. 1, 1933), pp. 1-383.

54. C. O. Lovejoy, M. J. Cohn, T. D. White, Proc. Natl. Acad. Sci. U.S.A. 96, 13247 (1999)

55. V. K. Sarin, G. M. Erickson, N. J. Giori, A. G. Bergman, D. R. Carter, Anat. Rec. 257, 174 (1999).

56. W. L. Straus Jr., Q. Rev. Biol. 5, 261 (1930)

57. D. N. Gomberg, J. Hum. Evol. 14, 553 (1985).

58. H. Gray, Gray's Anatomy (Lea and Febiger, Philadelphia, ed. 26,2008 ).

59. Supported by NSF grants $8210897,9318698,9512534$, 9632389, 9729060, 9910344, and 0321893 HOMINID$\mathrm{RHOI}$, and by the Japan Society for the Promotion of Science. We thank the Ministry of Tourism and Culture, the Authority for Research and Conservation of the Cultural Heritage, and the National Museum of Ethiopia for permissions and facilitation; the Afar Regional Government, the Afar people of the Middle Awash, and many other field workers for contributing directly to the data; the National Museum of Ethiopia, National Museums of Kenya, Transvaal Museum South Africa, Cleveland Museum of Natural History, Royal Museum of Central Africa Tervuren for access to comparative materials; L. Spurlock and P. L. Reno for assistance with dissections and histology preparations; D. Kubo and $\mathrm{H}$. Fukase for assistance in computed tomography (CT) scanning; C. Hernandez for calculation of section modulus data for Mohr's Circle analyses; M. Brunet, C. V. Ward, and ]. DeSilva for cooperation with comparative data; R. Meind for statistical advice and assistance; ]. DeSilva, P. L. Reno, M. A. Serrat, M. A. McCollum, M. Selby, A. Ruth, L. Jellema, S. W. Simpson, and B. A. Rosenman for aid in data collection and exceptionally helpful discussions; and H. Gilbert, ]. Carlson, and K. Brudvik for figure preparation.

\section{Supporting Online Material}

www.sciencemag.org/cgi/content/full/326/5949/72/DC1

Figs. S1 to S14

Tables S1 and S2

References

4 May 2009; accepted 14 August 2009

$10.1126 /$ science. 1175832 